\title{
COMMON POSITIONING AND TECHNICAL ERRORS IN PANORAMIC RADIOGRAPHY
}

\author{
S Pandey ${ }^{1 *}, \mathrm{KM}$ Pai $^{2}$, A Dhakal ${ }^{3}$ \\ ${ }^{1}$ Department of Oral Medicine and Radiology, Chitwan Medical Mollege,Bharatpur 10, Nepal. \\ ${ }^{2}$ Department of Oral Medicine and Radiology, Manipal College of Dental Sciences, Manipal University. \\ ${ }^{3}$ Department of Clinical Anatomy, Chitwan Medical College, Bharatp[ur 10, Nepal. \\ *Correspondence to: Dr Sushma Pandey, Department of Oral Medicine \& Radiology, Chitwan Medical College, Bharatpur 10, Nepal. \\ Email: pandeysusma@gmail.com
}

\begin{abstract}
Panoramic radiograph is one of the routinely used investigations in dentistry. The value of panoramic radiograph is reduced when they are of poor diagnostic quality. Therefore, the aim of the study is to be aware of the common positional and technical errors so as to minimize such errors occurring in the department. Panoramic radiographs of all the patients, who were taking radiographs for their own diagnostic purpose, were examined. All Radiographs taken for a 3 months period were 1010. All panoramic radiographs examined for various errors. Data were analyzed for the frequency of some common faults, both technical and processing errors, which directly contributed to failure of the radiographs. Total 1010 radiographs were analyzed for errors. $27.5 \%(n=278)$ were showing errors which ranged from technical errors $11.3 \%(n=14)$ to positional errors $16.2 \%(\mathrm{n}=164)$ and $72.5 \%$ of radiographs were error free. Most common technical error was density/dark radiographs which were $45 \%(n=51)$ and the most common positional error found was tongue not resting against the palate, $20 \%(n=32)$. Dark radiograph and tongue not resting against the palate were found common errors. Fewer errors are likely to be made when a quality assurance regimen and proper training, which includes the recognition and correction of errors, is followed.
\end{abstract}

Key Words: Panoramic radiograph, Positional error, Technical error.

\section{INTRODUCTION}

Panoramic radiograph is a simple extra-oral procedure that visualizes the entire maxillomandibular region on a single film. Since the introduction of panoramic radiography into general practice of dentistry in the early 1960's, it has gained considerable popularity as a diagnostic tool. The panoramic film is used alone or in combination with other radiographic views. The simplicity of operation, the broader scope of examination, ability to project anatomic structures and their intervening parts, as well as the low radiation dose are reasons for its continuously growing popularity. The equipment available for panoramic radiography has had rapid development during the last two decades. A variety of machines using different principles of $\mathrm{x}$-ray beam rotation are available for the dental profession. ${ }^{2}$ The value of panoramic radiograph is reduced when they are of poor diagnostic quality. This poor quality usually is not a result of an inherent limitation with the equipment but rather is a result of errors made by the operators during patient positioning and processing. ${ }^{1}$

Therefore, the aim of the study is to identify the most common positioning and technical errors in the panoramic radiographs taken in department of oral Medicine and Radiology, MCODS, Manipal. The data can be further used to evaluate frequency of positioning and technical error.

\section{MATERIAL AND METHODS}

This study was conducted in Department of Oral Medicine and Radiology, MCODS, Manipal over a period of 3 months between November 2009 and January 2010. Institutional Ethics Committee approval was taken from Kasturba Hospital, Manipal. Informed consent was taken from all the patients.

Panoramic radiographs of all the patients, who were taking radiographs for their own diagnostic purpose, were examined. Patient were positioned and OPG were taken by radiology technicians or post graduate students or faculty members on planmeca 2002cc panoramic machines. Then they were processed in promax processor and viewed under identical condition using a standard $15 \times 30$ light box in a room with subdued lighting. Radiographs were reported by the same person who has taken the radiograph to identify following panoramic errors as described by Langland. ${ }^{1}$

\section{Technical errors}

1. Low density radiograph:

2. High density and completely black image

3. Fogged Radiograph:

4. When the cassette is placed backward.

5. Only portion of film exposed

6. Vertical white line in Radiograph:

7. Alternating white and black vertical lines 
8. Random Artifacts

9. Processing problems
a. Streaking or uneven surface density
b. Static electricity
c. Smudge static marking
d. Glove smudge marking
e. folding mark

10. Fingerprints and Fluoride artifacts:

a. Dark grey fingerprints: film contaminated by the developer

b. White fingerprints: fixer contamination

c. Stanous fluoride: black fingerprint mark

\section{Crimp Marks}

\section{Positioning error}

12. If head is positioned too far forward

13. If head is positioned too far back and teeth not biting the groove

14. Midsagittal plane positioning errors
i. tilted head.
ii. off centered
iii. twisted
or any combination

15. Occlusal plane positioning error:

i. Downward over angulation of head

ii. Upward over angulation of head

16. Chin not rest on the chin support

17. Bite guide not used

18. Tongue not rest against palate

Data were statistically analysed using SPSS version 16 for the most commonly occurring positional and technical error with respect to above mentioned categories. Certain patients who have physical differences that prelude their being positioned properly at panoramic machine were excluded from the study.

\section{RESULTS}

This study was conducted in Department of Oral Medicine and Radiology, MCODS, Manipal. 1010 panoramic radiographs taken in three month period were evaluated.

As shown in Table 1 and Graph 1, 27.5\% $(n=278)$ were showing errors which ranged from technical errors $11.3 \%(\mathrm{n}=14)$ to positional errors $16.2 \%(n=164)$ and $72.5 \%$ of radiographs were error free.

Table 1: Prevalence of the different types of errors

\begin{tabular}{|l|l|l|}
\hline & Frequency & Prevalance \\
\hline Total Errors & 278 & $27.5 \%$ \\
\hline Technical Errors & 114 & $11.3 \%$ \\
\hline Positional Errors & 164 & $16.2 \%$ \\
\hline Total Radiograph & 1010 & \\
\hline
\end{tabular}

nadiographs without error $\square$ Technical errors $\square$ Positional errors

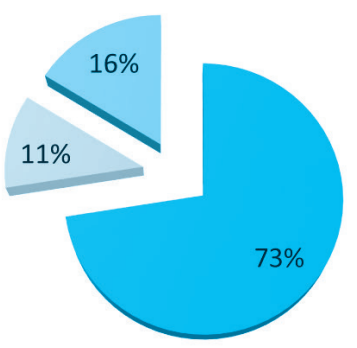

\section{Graph 1: Prevalence of technical and positional errors}

Table 2 and Graph 2 reveal frequency of some common technical errors, which were $45 \%(n=51)$ high density/dark radiographs, $38 \%(n=44 \%)$ were light radiographs, $10 \%(n=11)$ were processing problems, 3\% $(n=4)$ were partially exposed, $2 \%(n=2)$ were having random artifacts, $1 \%(n=1)$ were fogged radiograph and crimp marks.

Table 2: Frequency of different technical errors

\begin{tabular}{|c|c|c|}
\hline & Error 1 & \\
\hline Types & Frequency & Percentage \\
\hline $\mathbf{1}$ & 44 & 38 \\
\hline $\mathbf{2}$ & 51 & 45 \\
\hline $\mathbf{3}$ & 1 & 1 \\
\hline $\mathbf{5}$ & 4 & 3 \\
\hline $\mathbf{8}$ & 2 & 2 \\
\hline $\mathbf{9}$ & 11 & 10 \\
\hline $\mathbf{1 1}$ & 1 & 1 \\
\hline
\end{tabular}

$1=$ low density radiography/Light radiography, 2 = High density radiography/Dark radiography, 3= Fogged Radiography, 5= Only portion offilm exposed, $8=$ Random Artifacts, $9=$ Processing problems, $11=$ Crimp marks

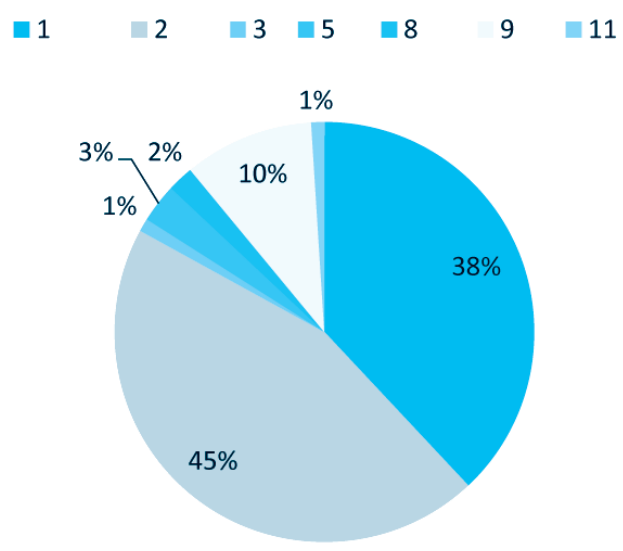

Graph 2: Percentage of some common technical errors 
Table 3 and Graph 3 reveal frequency of some commonly occurring positional errors. $23 \%(n=37)$ were tongue not resting against the palate, $20 \%(\mathrm{n}=32)$ were spinal column positioning error, $17 \%(n=28)$ were occlusal plane positioning error, $10 \%$ $(n=17)$ were having nose ring artifact, $9 \%(n=15)$ were distorted due to patient movement. 5\% $(n=9)$ were midsagittal plane positioning error and ear jewelery artifact, $2 \%$ were chin not resting on the chin support, lips open, head positioning too far forward and neck chain artifact. $1 \%(n=1)$ were bite guard not used, prostheses left in oral cavity and double exposure errors.

Table 3: Frequency of different positional errors

\begin{tabular}{|l|l|l|}
\hline & Error 2 & \\
\hline Error Type & Frequency & Percentage \\
\hline 12 & 3 & 2 \\
\hline 14 & 9 & 5 \\
\hline 15 & 28 & 17 \\
\hline 16 & 4 & 2 \\
\hline 17 & 1 & 1 \\
\hline 18 & 37 & 23 \\
\hline 19 & 4 & 2 \\
\hline 20 & 1 & 1 \\
\hline 22 & 3 & 2 \\
\hline 23 & 9 & 5 \\
\hline 24 & 17 & 10 \\
\hline 26 & 32 & 20 \\
\hline 28 & 15 & 9 \\
\hline 29 & 1 & 1 \\
\hline
\end{tabular}

$12=$ If head is positioned too far forward, 14=Midsagittal plane positioning errors, 15=Occlusal plane positioning error, 16=Chin not rest on the chin support, 17=Bite guide not used, 18=Tongue not rest against palate, 19= Lips open, $20=$ Prosthesis left in place, 22=Neck chain artefact, 23=Ear jewelery artefact, 22=Neck chain artefact, 24=Nose ring artefact, 26=Spinal column positioning error, 28=Distortion due to patient movement, 29=Double Exposure

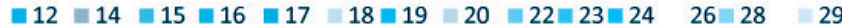

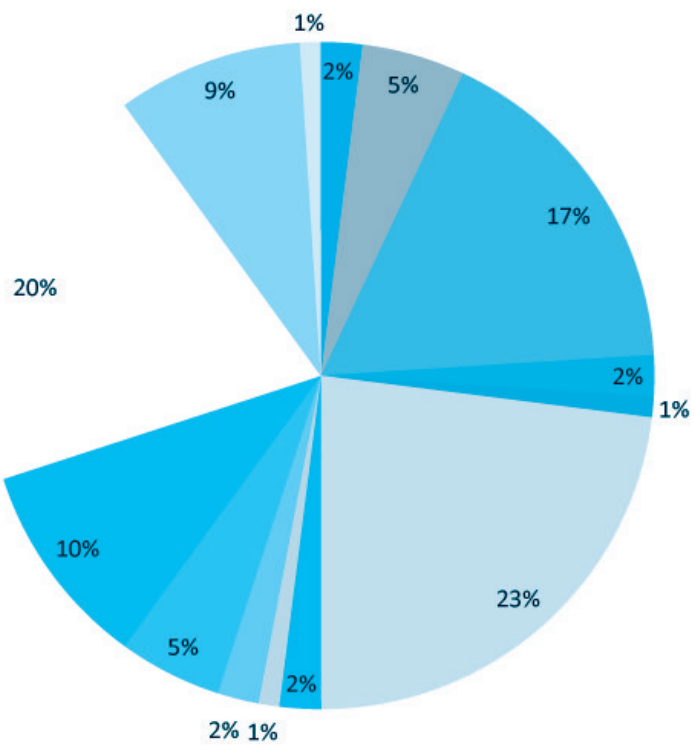

Graph 3: Percentage of some common positional errors

\section{DISCUSSION}

In our study various errors were observed on 1010 panoramic radiographs examined. Data were analyzed for the frequency of some common faults, both technical and processing errors, which directly contributed to failure of the radiographs.

In this study, the most common errors were related to patient positioning. This finding is in agreement with the findings of previous study. ${ }^{2,3}$ They also demonstrate that patient positioning errors were more common than technical errors. One of the reasons for this could be the inability of some patient to follow the instruction made by radiographers.

Among positional error, the tongue not positioning correctly in the palate during exposure was the most common error identified; this result is in agreement with the result obtained in the studies done by other investigators..$^{2,4}$ The possible explanation for this error may be a lack of communication between the operator and the patients because of different languages. The technician may find difficulty to instruct the patients to swallow and to keep the tongue on the roof of the mouth. Another explanation is that the patients sometime may misunderstand the instructions, putting only the tip of the tongue on the palate, or the patients do not pay much attention to the instruction given by the operator. ${ }^{8}$ Among technical errors, low density radiographs were common. This finding is in agreement with the study done by Ruston et al. ${ }^{4}$ This may have been caused by underexposure, not adjusting the parameters according to patient morphology or it was largely related to under-development. Careful attention to processing quality control, including timely replacement of chemicals, time 
and temperature control and automatic processor maintenance, adjusting adequate equipment set up, can do much to avoid density and contrast errors. The use of digital radiography could also eliminate this problem.

Hence fewer errors are likely to be made when a quality assurance regimen and proper training, which includes the recognition and correction of errors, is followed.

\section{REFERENCES}

1. Langland OE, Langlais RP, Morris CR. Principles and practice of panoramic radiology. Philadelphia, W.B. Saunders Co 1982;84-156.
2. Schiff T, D Ambrosio J, Glass BJ, Langlais RP, McDavid WD. Common positioning and technical errors in panoramic radiography. J Am Dent Assoc 1986;113:422-426.

3. Farzaneh K, Masume J, Farzad E. Evaluation of Panoramic Radiograph Errors, JODDD. 2008;2:3.

4. Rushton VE, Horner K. The use of panoramic radiology in dental practice. J Dent 1996;24(3):185-20.

5. Natalia A. Brezden BS, Sharon L. Brooks, Ann Arbor, Mich. Evaluation of panoramic dental radiographs taken in private practice. Oral surg. Oral med, Oral Patho1987;63:617-21. 